\title{
Quantum Detailed Balance and KMS Condition
}

\author{
Andrzej Kossakowski \\ Institute of Physics, N. Copernicus University, Toruń, Poland \\ Alberto Frigerio ${ }^{\star}$ and Vittorio Gorini \\ Istituto di Fisica dell' Università, Milano, and \\ Istituto Nazionale di Fisica Nucleare, Sezione di Milano, Milano, Italy

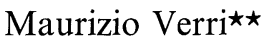 \\ Istituto di Matematica del Politecnico, Milano, and \\ Istituto Nazionale di Fisica Nucleare, Sezione di Milano, Milano, Italy
}

\begin{abstract}
A definition of detailed balance for quantum dynamical semigroups is given, and its close connection with the KMS condition is investigated.
\end{abstract}

\section{Introduction}

In recent works [1-3] various definitions of detailed balance for a quantum Markovian master equation have been proposed and discussed. In this paper, we give a definition of detailed balance for a quantum dynamical semigroup of a $W^{*}$ algebra, which extends the analogous notion proposed in [3] for quantum dynamical semigroups of matrix algebras (for a heuristic motivation, based on the analogy with the corresponding classical concept, see $[3,4])$. We give the general form of the generator $L$ of a dynamical semigroup of $\mathscr{B}(\mathscr{H})$ satisfying detailed balance and with a norm continuous dissipative part, thus extending the result of [3].

The physical meaning of this seemingly formal definition is investigated by showing that the property of detailed balance is characteristic of dynamical semigroups describing relaxation to thermal equilibrium, thus providing yet another characterization of KMS states.

\section{Quantum Detailed Balance}

Let $\mathscr{M}$ be a $W^{*}$-algebra. A dynamical semigroup of $\mathscr{M}$ [5-7] is a weakly *continuous one-parameter semigroup $\left\{\Phi_{t}: t \geqq 0\right\}$ of completely positive identity preserving normal maps of $\mathscr{M}$ into itself, with $\Phi_{0}$ the identity map.

Let $\varrho$ be a faithful normal state on $\mathscr{M}$ which is stationary under $\left\{\Phi_{t}\right\}$, and denote by $(\mathscr{K}, \pi, \Omega)$ the GNS triple associated to $\varrho$. There exists $[8,9]$ a strongly continuous contraction semigroup $\left\{\hat{\Phi}_{t}\right\}$ on $\mathscr{K}$ such that

$$
\hat{\Phi}_{t} \pi(A) \Omega=\pi\left(\Phi_{t}(A)\right) \Omega \quad \text { for all } \quad A \in \mathscr{M}, \quad t \geqq 0 .
$$

$\star$ A fellowship from the Italian Ministry of Public Education is acknowledged

$\star \star$ A fellowship from the Italian National Research Council is acknowledged 
Denote by $\hat{L}$ the densely defined generator of $\left\{\hat{\Phi}_{t}\right\}$.

Definition. We say that $\left\{\Phi_{t}\right\}$ satisfies detailed balance w.r.t. $\varrho$ if there exist a selfadjoint operator $\hat{L}_{s}$ and a skewadjoint operator $\hat{L}_{h}$ in $\mathscr{K}$ such that

(i) $\hat{L} \psi=\hat{L}_{s} \psi+\hat{L}_{h} \psi$

for all $\psi$ in a dense linear manifold $\mathscr{C} \subset \mathscr{K}$ which is a common core for $\hat{L}, \hat{L}_{s}, \hat{L}_{h}$;

(ii) $\pi\left(\alpha_{t}(A)\right) \Omega=\exp \left(\hat{L}_{h} t\right) \pi(A) \Omega$

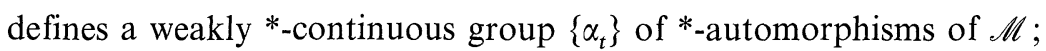

(iii) $\pi\left(\Gamma_{t}(A)\right) \Omega=\exp \left(\hat{L}_{s} t\right) \pi(A) \Omega$

defined a dynamical semigroup $\left\{\Gamma_{t}\right\}$ of $\mathscr{M}$. $\left\{\Phi_{t}\right\}$.

$\left\{\alpha_{t}\right\}$ (resp., $\left\{\Gamma_{t}\right\}$ ) will be called the Hamiltonian part (resp., the dissipative part) of

Remarks. It can be shown that if a decomposition (i) exists with $\hat{L}_{s}=\hat{L}_{s}^{*}, \hat{L}_{h}=-\hat{L}_{h}^{*}$, then it is unique (Appendix A) and that (iii) is actually a consequence of (i) and (ii) (Appendix B).

The state $\varrho$ is stationary for $\left\{\alpha_{t}\right\}$ and $\left\{\Gamma_{t}\right\}$. Indeed, $\varrho\left(\alpha_{t}(A)\right)=\varrho\left(\alpha_{-t}(\mathbb{1}) A\right)=\varrho(A)$ and $\varrho\left(\Gamma_{t}(A)\right)=\varrho\left(\Gamma_{t}(\mathbb{1}) A\right)=\varrho(A)$.

Both $\left\{\alpha_{t}\right\}$ and $\left\{\Gamma_{t}\right\}$ commute with the modular automorphism group $\left\{\sigma_{t}\right\}[10]$ associated to $\varrho$. This property, which for $\left\{\alpha_{t}\right\}$ is well known [11], is a special case of the following

Proposition 2.1. If two completely positive identity preserving maps $\Gamma$ and $\Gamma^{\prime}$ of a $W^{*}$ algebra $\mathscr{M}$ satisfy

$$
\varrho\left(\Gamma^{\prime}(A) B\right)=\varrho(A \Gamma(B)) \text { for all } A, B \in \mathscr{M},
$$

where $\varrho$ is a faithful normal state on $\mathscr{M}$, then $\Gamma$ and $\Gamma^{\prime}$ commute with $\left\{\sigma_{t}\right\}$.

Sketch of the Proof. The contraction maps $\hat{\Gamma}$ and $\hat{\Gamma}^{\prime}$ on $\mathscr{K}$, associated to $\Gamma$ and $\Gamma^{\prime}$ as in Equation (2.1), commute strongly with the modular operator $\Delta$ [12, Lemma 2$].$ Since $\Omega$ is separating for $\pi(\mathscr{M})$, the statement follows.

In the following, we restrict our consideration to the case $\mathscr{M}=\mathscr{B}(\mathscr{H})$, the algebra of all bounded operators on a separable Hilbert space $\mathscr{H}$. This is a suitable framework for the description of the reduced dynamics of a spatially confined quantum open system. For this case, we give a classification of the generators of dynamical semigroups which satisfy detailed balance and possess a norm continuous dissipative part.

The Hamiltonian part $\left\{\alpha_{t}\right\}$ of a dynamical semigroup $\left\{\Phi_{t}\right\}$ of $\mathscr{B}(\mathscr{H})$ satisfying detailed balance with respect to a faithful normal state $\varrho$ is of the form $[13$, Corollary 2.9.32, p. 120 and Remark 2, p. 166]

$$
\alpha_{t}(A)=\exp (i t H) A \exp (-i t H), \quad A \in \mathscr{B}(\mathscr{H}),
$$

where $H$ is a selfadjoint operator in $\mathscr{H}$, commuting with the density matrix which expresses $\varrho$ (and for which we shall use the same notation $\varrho$ ). 
If the dissipative part $\left\{\Gamma_{t}\right\}$ of $\left\{\Phi_{t}\right\}$ is norm continuous, then $\Gamma_{t}=\exp \left(L_{s} t\right)$, where $L_{s}$ is a bounded linear map of $\mathscr{B}(\mathscr{H})$ into itself. In this case, $\pi(\mathscr{B}(\mathscr{H})) \Omega$ is a core for $\hat{L}_{s}$ [14, Theorem X.49], and the restriction of $\hat{L}_{s}$ to $\pi(\mathscr{B}(\mathscr{H}))$ is given by

$$
\hat{L}_{s} \pi(A) \Omega=\pi\left(L_{s}(A)\right) \Omega, \quad A \in \mathscr{B}(\mathscr{H}) .
$$

Hence the selfadjointness of $\hat{L}_{s}$ is equivalent to

$$
\varrho\left(L_{s}(A) B\right)=\varrho\left(A L_{s}(B)\right) \quad \text { for all } \quad A, B \in \mathscr{B}(\mathscr{H}) .
$$

A linear map $L_{s}$ of $\mathscr{B}(\mathscr{H})$ satisfying (2.6) will be called $\varrho$-symmetric ; a linear map $L_{a}$ of $\mathscr{B}(\mathscr{H})$ will be called $\varrho$-antisymmetric if

$$
\varrho\left(L_{a}(A) B\right)=-\varrho\left(A L_{a}(B)\right) \quad \text { for all } \quad A, B \in \mathscr{B}(\mathscr{H}) .
$$

Theorem 2.2. The general form of a $\varrho$-symmetric generator $L_{s}$ of a norm continuous dynamical semigroup $\left\{\Gamma_{t}\right\}$ of $\mathscr{B}(\mathscr{H})$ is the following

$$
L_{s}(A)=\underset{N \rightarrow \infty}{u}-\lim \sum_{r r^{\prime} s s^{\prime}=1}^{N} C_{r r^{\prime} s s^{\prime}}\left[P_{r r^{\prime}} A P_{s^{\prime} s}-\frac{1}{2}\left\{P_{r r^{\prime}} P_{s^{\prime} s}, A\right\}\right],
$$

where $A \in \mathscr{B}(\mathscr{H})$ and

$P_{r r^{\prime}}=|r\rangle\left\langle r^{\prime}\right|,\{|r\rangle\}$ a complete orthonormal set (c.o.n.s.)

of eigenvectors of $\varrho, \varrho|r\rangle=\varrho_{r}|r\rangle$;

$\left\{C_{r r^{\prime} s^{\prime}}\right\}$ is a positive matrix in the sense that

$\sum_{r r^{\prime} s s^{\prime}} \overline{x_{r r^{\prime}}} C_{r r^{\prime} s s^{\prime}} x_{s s^{\prime}} \geqq 0 \quad$ for all sequences

$\left\{x_{r r^{\prime}}\right\}$ for which the expression converges;

$$
\begin{aligned}
& \sum_{r r^{\prime} s s^{\prime}=1}^{N} C_{r r^{\prime} s s^{\prime}} P_{r r^{\prime}} P_{s^{\prime} s} \text { converges ultraweakly as } N \rightarrow \infty ; \\
& C_{r r^{\prime} s s^{\prime}} \varrho_{s}=C_{s^{\prime} s r^{\prime} r} \varrho_{s^{\prime}} \\
& \left(\text { or, equivalently, } C_{r r^{\prime} s s^{\prime}} \varrho_{r}=C_{s^{\prime} s r^{\prime} r} \varrho_{r^{\prime}}\right) .
\end{aligned}
$$

Remark. Note that the condition characterizing $\varrho$-symmetry is $(2.12)$.

Proof. $L_{s}$ can be written as [7]

$$
L_{s}(A)=\Psi(A)-\frac{1}{2}\{\Psi(\mathbb{1}), A\}+i[H, A], \quad A \in \mathscr{B}(\mathscr{H}),
$$

where $\Psi$ is a completely positive normal map of $\mathscr{B}(\mathscr{H})$ and $H=H^{*} \in \mathscr{B}(\mathscr{H})$. By Proposition 2.1, $L_{s}$ commutes with $\sigma_{t}=\varrho^{i t}(.) \varrho^{-i t}$. Hence, with no loss of generality, we can assume $\Psi$ to commute with $\left\{\sigma_{t}\right\}$ and $H$ to commute with $\varrho$. Indeed, $\Psi$ can be replaced by $\lim _{T \rightarrow \infty}(1 / 2 T) \int_{-T}^{T} d t \sigma_{t} \Psi \sigma_{-t}$, where the limit exists and defines a normal map (compare [15]) and the convergence is in the bounded-weak topology, with respect to which the cone of completely positive maps is closed [16]. Similarly, $H$ can be replaced by $\underset{T \rightarrow \infty}{u}-\lim _{T \rightarrow \infty}(1 / 2 T) \int_{-T}^{T} d t \sigma_{t}(H)$ (compare [15]). We can choose $\{|r\rangle\}$ 

such that $H|r\rangle=\varepsilon_{r}|r\rangle$. Write $\Psi(A)=\sum_{j} V_{j}^{*} A V_{j}, A, V_{j} \in \mathscr{B}(\mathscr{H})$ (ultraweak convergence)
[17] and let

$$
K_{r r^{\prime} s s^{\prime}}=\operatorname{Tr}\left[P_{s r} \Psi\left(P_{r^{\prime} s^{\prime}}\right)\right]=\sum_{j}\left\langle r\left|V_{j}^{*}\right| r^{\prime}\right\rangle\left\langle s^{\prime}\left|V_{j}\right| s\right\rangle .
$$

Since $\Psi$ commutes with $\left\{\sigma_{t}\right\}, K_{r r^{\prime} s s^{\prime}}=0=K_{s^{\prime} r^{\prime} r}$ unless $\varrho_{s} \varrho_{s^{\prime}}^{-1}=\varrho_{r} \varrho_{r^{\prime}}^{-1}$. Then the $\varrho$ symmetry of $L_{s}$, i.e.

$$
\operatorname{Tr}\left[\varrho P_{s r} L_{s}\left(P_{r^{\prime} s^{\prime}}\right)\right]=\operatorname{Tr}\left[\varrho L_{s}\left(P_{s r}\right) P_{s^{\prime} r^{\prime}}\right] \quad \text { for all } r, r^{\prime}, s, s^{\prime}
$$

is equivalent to

$$
\varrho_{s} K_{r r^{\prime} s s^{\prime}}-\varrho_{s^{\prime}} K_{s^{\prime} s r^{\prime} r}=2 i\left(\varepsilon_{s}-\varepsilon_{r}\right) \varrho_{s} \delta_{r r^{\prime}} \delta_{s s^{\prime}}
$$

Let $H_{N}=\sum_{r=1}^{N} \varepsilon_{r} P_{r r}$. We may set $\varepsilon_{1}=0$; then, by Equation (2.13),

$$
H_{N}=(1 / 2 i) \sum_{r r^{\prime}=1}^{N}\left(K_{11 r^{\prime} r}-K_{r r^{\prime} 11}\right) P_{r r^{\prime}}
$$

Let $\Psi_{N}(A)=\sum_{r r^{\prime} s s^{\prime}=1}^{N} K_{r r^{\prime} s s^{\prime}} P_{r r^{\prime}} A P_{s^{\prime} s}, \quad A \in \mathscr{B}(\mathscr{H})$. Defining the completely positive $\operatorname{map} E_{N}: \mathscr{B}(\mathscr{H}) \rightarrow \mathscr{B}(\mathscr{H})$ by

$$
E_{N}(A)=Q_{N} A Q_{N}, \quad Q_{N}=\sum_{r=1}^{N} P_{r r},
$$

we have $H_{N}=E_{N}(H)$ and $\Psi_{N}=E_{N} \Psi E_{N}$.

Define also the completely positive map $\Psi_{N}^{\prime}$ of $\mathscr{B}(\mathscr{H})$ by

$$
\begin{aligned}
\Psi_{N}^{\prime}(A) & =\sum_{j} E_{N}\left(V_{j}^{*}-\bar{v}_{j} \mathbb{1}\right) A E_{N}\left(V_{j}-v_{j} \mathbb{1}\right) \\
& =\sum_{r r^{\prime} s s^{\prime}=1}^{N} C_{r r^{\prime} s s^{\prime}} P_{r r^{\prime}} A P_{s^{\prime} s}
\end{aligned}
$$

where $v_{j} \equiv\left\langle 1\left|V_{j}\right| 1\right\rangle$ and

$$
\begin{aligned}
C_{r r^{\prime} s s^{\prime}} & \left.=\sum_{j}\left\langle r\left|\left(V_{j}^{*}-\overline{v_{j}} \mathbb{1}\right)\right| r^{\prime}\right\rangle\left\langle s^{\prime}\right| V_{j}-v_{j} \mathbb{1}\right)|s\rangle \\
& =K_{r r^{\prime} s s^{\prime}}-\delta_{r r^{\prime}} K_{11 s s^{\prime}}-\delta_{s s^{\prime}} K_{r r^{\prime} 11}+\delta_{r r^{\prime}} \delta_{s s^{\prime}} K_{1111} .
\end{aligned}
$$

$\left\{C_{r r^{\prime} s s^{\prime}}\right\}$ is positive by definition and satisfies condition (2.12) by Equation (2.13). Moreover, we have

$$
\begin{aligned}
\sum_{r r^{\prime} s s^{\prime}=1}^{N} C_{r r^{\prime} s s^{\prime}} P_{r r^{\prime}} P_{s^{\prime} s} & =\Psi_{N}^{\prime}(\mathbb{1}) \\
& =E_{N} \Psi\left(Q_{N}\right)-2 i E_{N}(H)-E_{N}(\mathbb{1}) K_{1111} .
\end{aligned}
$$

Now $\underset{N \rightarrow \infty}{u w-\lim _{N}} E_{N}(H)=H, \quad u w-\lim _{N \rightarrow \infty} E_{N}(\mathbb{1})=\mathbb{1}$, and $\lim _{N \rightarrow \infty} \operatorname{Tr}\left\{A_{0}\left[E_{N} \Psi\left(Q_{N}\right)-\Psi(\mathbb{1})\right]\right\}=0$ for all $A_{0}$ in the linear span $\mathscr{B}_{0}$ of the $P_{r s}$ 's. Since $\mathscr{B}_{0}$ is dense in the space of trace class operators on $\mathscr{H}$ and $\operatorname{Tr}\left\{A_{0}\left[E_{N} \Psi\left(Q_{N}\right)-\Psi(\mathbb{1})\right]\right\}$ is bounded by $2\left\|A_{0}\right\|_{1}\|\Psi\|$, the 
expression (2.14) converges ultraweakly as $N \rightarrow \infty$ to

$$
\Psi(\mathbb{1})-2 i H-K_{1111} \mathbb{1} .
$$

Finally, a straightforward calculation yields

$$
\begin{aligned}
& \Psi_{N}^{\prime}(A)-\frac{1}{2}\left\{\Psi_{N}^{\prime}(\mathbb{1}), E_{N}(A)\right\} \\
& =\Psi_{N}-\frac{1}{2}\left\{\Psi_{N}(\mathbb{1}), E_{N}(A)\right\}+i\left[H_{N}, E_{N}(A)\right] \\
& =E_{N} L E_{N}(A)+\frac{1}{2}\left\{E_{N}\left(\Psi\left(\mathbb{1}-Q_{N}\right)\right), E_{N}(A)\right\}, \quad A \in \mathscr{B}(\mathscr{H}) .
\end{aligned}
$$

For $A$ in $\mathscr{B}_{0}$, the ultraweak limit of the r.h.s. as $N \rightarrow \infty$ is $L(A)$ by the same arguments as above. The extension to $\mathscr{B}(\mathscr{H})$ is made using the ultraweak density of $\mathscr{B}_{0}$ in $\mathscr{B}(\mathscr{H})$, the ultraweak continuity of $L_{s}$ and uniform boundedness estimates.

Conversely, if a map $L_{s}$ is of the form (2.8) and satisfies (2.9)-(2.11), then it is of the Lindblad type [7] with no Hamiltonian part, and

$$
\Psi(A)=u w-\lim _{N \rightarrow \infty} \sum_{r r^{\prime} s s^{\prime}=1}^{N} C_{r r^{\prime} s s^{\prime}} P_{r r^{\prime}} A P_{s^{\prime} s}, \quad A \in \mathscr{B}(\mathscr{H}) .
$$

By Equation (2.11), $\Psi$ is well defined and normal. It is completely positive by Equation (2.10) and the fact that the cone of completely positive maps is closed in the bounded-weak topology [16]. The $\varrho$-symmetry of $L_{s}$ follows immediately from Equation (2.12). Q.E.D.

Remark. In the special case $\operatorname{dim} \mathscr{H}=N<\infty$ and $\varrho$ the central state $\varrho=\tau \equiv N^{-1} \mathbb{1}$, it follows from the form of the generator given in [6] that a $\tau$-symmetric generator has the general form

$$
L_{s}(A)=\frac{1}{2} \sum_{i j=1}^{N^{2}-1} c_{i j}\left\{F_{j}\left[A, F_{i}\right]+\left[F_{j}, A\right] F_{i}\right\},
$$

where $F_{j}=F_{j}^{*}, \operatorname{Tr}\left(F_{j}\right)=0, \operatorname{Tr}\left(F_{i} F_{j}\right)=\delta_{i j}$, and where $\left\{c_{i j}\right\}$ is a positive symmetric matrix. These are exactly the generators describing the dynamics of an $N$-level system with a purely random Gaussian stochastic Hamiltonian [18], or the reduced dynamics of an $\mathrm{N}$-level system in the limit of singular coupling to a reservoir at infinite temperature [19].

Remark. An elegant expression of the general form of a $\varrho$-symmetric generator has been given by Alicki [3] in the case when $\operatorname{dim} \mathscr{H}=N<\infty$ and the spectrum of $\varrho$ is nondegenerate. The latter restriction can be dropped with the aid of the result (2.15) above, and the form of $L_{s}$ then reads

where

$$
L_{s}(A)=\frac{1}{2} \sum_{i j=1}^{N} D_{i j}\left\{X_{i j}^{*}\left[A, X_{i j}\right]+\left[X_{i j}^{*}, A\right] X_{i j}\right\},
$$

$$
\begin{aligned}
& \operatorname{Tr}\left(X_{i j}^{*} X_{k l}\right)=\delta_{i k} \delta_{j l}, \\
& \varrho X_{i j} \varrho^{-1}=\varrho_{i} \varrho_{j}^{-1} X_{i j}, \\
& X_{i j}^{*}=X_{i j} \text { for } \varrho_{i}=\varrho_{j} ; \quad X_{i j}^{*}=X_{j i} \text { for } \varrho_{i} \neq \varrho_{j}, \\
& D_{i j} \geqq 0 \text { for all } i, j ; \quad D_{i j} \varrho_{j}=D_{j i} \varrho_{i} \text { for } \varrho_{i} \neq \varrho_{j} .
\end{aligned}
$$

The proof of this statement is given in Appendix C. 


\section{Weak Coupling Limit and KMS Condition}

In this section, we investigate the close connection between quantum detailed balance for dynamical semigroups obtained in the limit of weak coupling [20] and the KMS condition for the associated reservoirs.

Consider a spatially confined quantum system $S$ interacting with an infinite reservoir $R$. We describe $S$ by an algebra of observables $\mathscr{B}(\mathscr{H}), \mathscr{H}$ a separable Hilbert space, with dynamics determined by a semibounded selfadjoint Hamiltonian $H$ with pure point spectrum and such that $\exp (-\beta H)$ is trace class for all $\beta>0$.

We denote by $\mathscr{A}$ the $C^{*}$-algebra of observables of $R$ and assume $R$ to be initially in a state $\omega$ which is stationary under the free evolution. We describe the latter by a weakly *-continuous group $\left\{\alpha_{t}\right\}$ of *-automorphisms of the von Neumann algebra $\mathscr{A}_{\omega}=\pi_{\omega}(\mathscr{A})^{\prime \prime}$, implemented by a group of unitaries of the GNS space $\mathscr{H}_{\omega}$, assumed to be separable. In the following, these conditions will be summarized by the notation $R=\left(\mathscr{A}, \omega, \alpha_{t}\right)$.

We couple $S$ to $R$ by a bounded interaction

$$
g V=g \sum_{j=1}^{n} F_{j} \otimes \varphi_{j}
$$

with $g>0, F_{j}=F_{j}^{*} \in \mathscr{B}(\mathscr{H}), \varphi_{j}=\varphi_{j}^{*} \in \mathscr{A}_{\omega}, \omega\left(\varphi_{j}\right)=0$.

Under suitable conditions on the sufficiently fast decay of the multi-time correlation functions of the operators $\varphi_{j}$ in the state $\omega$ [20, Theorem 2.3], the reduced dynamics in the interaction picture for the observables of $S$ in the weak coupling limit $\left(g \rightarrow 0, \tau=g^{2} t\right.$ finite) is described by a dynamical semigroup whose generator $L$ is bounded, commutes with the free evolution of $S$ and is given by [20]

$$
\begin{aligned}
& L(A)=\underset{N \rightarrow \infty}{u w-\lim _{N}} \sum_{\substack{r r^{\prime} s^{\prime}=1 \\
\varepsilon_{r}-\varepsilon_{r^{\prime}}=\varepsilon_{s}-\varepsilon_{s^{\prime}}}}^{N} \sum_{i j=1}^{n}\left(F_{j}\right)_{r^{\prime}}\left(F_{i}\right)_{s^{\prime} s}\left(i s_{i j}\left(\varepsilon_{r}-\varepsilon_{r^{\prime}}\right) \delta_{r^{\prime} s^{\prime}}\left[P_{r s^{\prime}}, A\right]\right. \\
& \left.+\hat{h}_{i j}\left(\varepsilon_{r}-\varepsilon_{r^{\prime}}\right)\left[P_{r r^{\prime}} A P_{s^{\prime} s}-\frac{1}{2}\left\{P_{r r^{\prime}} P_{s^{\prime} s}, A\right\}\right]\right),
\end{aligned}
$$

where $A \in \mathscr{B}(\mathscr{H})$ and

$$
\begin{aligned}
P_{r r^{\prime}} & =|r\rangle\left\langle r^{\prime}\right|,\{|r\rangle\} \text { a c.o.n.s. of eigenvectors of } H, \\
H|r\rangle & =\varepsilon_{r}|r\rangle, \quad\left(F_{j}\right)_{r r^{\prime}} \equiv\left\langle r\left|F_{j}\right| r^{\prime}\right\rangle ; \\
\hat{h}_{i j}(\lambda) & =\int_{-\infty}^{+\infty} d t e^{-i \lambda t} h_{i j}(t), \quad h_{i j}(t) \equiv \omega\left(\varphi_{j} \alpha_{t}\left(\varphi_{i}\right)\right) ; \\
s_{i j}(\lambda) & =i \int_{0}^{+\infty} d t e^{-i \lambda t} h_{i j}(t)-\frac{i}{2} \hat{h}_{i j}(\lambda) \\
& =\frac{1}{2 \pi} \mathscr{P} \int_{-\infty}^{+\infty} d u \frac{\hat{h}_{i j}(u)}{u-\lambda},
\end{aligned}
$$

$\mathscr{P}$ denoting the principal part. By virtue of Davies' conditions [20] the $\hat{h}_{i j}(\lambda)$ are continuous functions. Furthermore, $\left\{\hat{h}_{i j}(\lambda)\right\}$ is a positive matrix for all $\lambda \in \mathbb{R}$ by Bochner's theorem [14, Theorem IX.9].

The generator $L$ is the sum of a Hamiltonian part $i\left[H_{1},.\right]$, where $H_{1}$ commutes with $H$, and a non-Hamiltonian part which depends on the correlation functions 
$\hat{h}_{i j}(\lambda)$ of $R$. If $\omega$ is a KMS state at inverse temperature $\beta>0$, then $\varrho_{\beta}=\exp (-\beta H) / \operatorname{Tr}[\exp (-\beta H)]$ is a stationary state for the reduced dynamics $\{\exp (L t)\}$, the Hamiltonian part is $\varrho_{\beta}$-antisymmetric, and the non-Hamiltonian part is $\varrho_{\beta}$-symmetric as a consequence of the KMS condition on the Fourier transforms

$$
\hat{h}_{j i}(-\lambda)=\exp (-\beta \lambda) \hat{h}_{i j}(\lambda) \text { for all } \lambda \in \mathbb{R} .
$$

Then, $\{\exp (L t)\}$ satisfies detailed balance with respect to the canonical state $\varrho_{\beta}$. Negative or infinite temperatures $(\beta \leqq 0)$ are allowed if $S$ is an $N$-level system.

Detailed balance for the Markov process induced on the diagonal elements was already recognized by Davies [20].

In order to prove the converse result that detailed balance for the reduced dynamics implies the KMS condition for the reservoir, we need some technical assumptions.

We require that there exists a subset $\mathscr{R}$ of the selfadjoint part of $\mathscr{A}_{\omega}$ such that

a) if $\varphi$ is in $\mathscr{R}$, then also $\alpha_{t}(\varphi)$ is in $\mathscr{R}$ for all $t$, and the smeared operators $\varphi(f) \equiv \int d t f(t) \alpha_{t}(\varphi)$ are in $\mathscr{R}$ for all functions $f(t)$ whose Fourier transforms are $C^{\infty}$ of compact support;

b) if $\varphi$ is in $\mathscr{R}$, then $\omega(\varphi)=0$, and for all $\varphi_{i}, \varphi_{j}, h_{i j}(t) \equiv \omega\left(\varphi_{j} \alpha_{t}\left(\varphi_{i}\right)\right)$ is in $L^{1}(\mathbb{R})$, so that its Fourier transform $\hat{h}_{i j}(\lambda)$ is continuous;

c) the multi-time correlation functions of the operators $\varphi_{j} \in \mathscr{R}$ in the state $\omega$ satisfy the conditions which allow the application of Theorem 2.3 of [20], so that the weak coupling limit technique can be applied and leads to the result (3.2) for all couplings of the form (3.1) with $\varphi_{j} \in \mathscr{R}$;

d) for any bounded interval $I \subset \mathbb{R}$, there exists a finite collection $\left\{\varphi_{j}\right\}_{j=1}^{n} \subset \mathscr{R}$ such that

$$
\sum_{i j=1}^{n} \hat{h}_{i j}(\lambda) \neq 0 \quad \text { for all } \quad \lambda \in I .
$$

If $R$ is a quasifree fermion reservoir and $\omega$ is a quasifree gauge invariant state, we can choose $\mathscr{R}$ to be the set of selfadjoint linear combinations of creation and annihilation operators smeared with test functions having a compact support in the energy space; then, all assumptions a),..., d) are satisfied (for an explicit form of condition c) in the case of a quasifree reservoir, see Section 3 of [20]). For general reservoirs, we lack an explicit form of condition c), and we need either one of the following additional conditions to hold:

e) the linear span of $\mathscr{R} \cup\{\mathbb{1}\}$ is a strongly dense *-subalgebra of $\mathscr{A}_{\omega}$;

$\left.\mathrm{e}^{\prime}\right)$ the dynamics $\left\{\alpha_{t}\right\}$ can be defined at the $C^{*}$-algebra level as a strongly continuous group of $*$-automorphisms of $\mathscr{A}$ and the linear span of $\mathscr{R} \cup\{\mathbb{1}\}$ is uniformly dense in $\mathscr{A}$.

Theorem 3.1. Let $R=\left(\mathscr{A}, \omega, \alpha_{t}\right)$ be an infinite quantum system coupled to a spatially confined quantum system $S$ with $\operatorname{dim} \mathscr{H} \geqq 6$. Assume that the reduced dynamics $\{\exp (L t)\}$ of $S$ in the weak coupling limit satisfies detailed balance with respect to some faithful state $\varrho$, for all choices of $H$ and of a coupling $g V$ of the form (3.1), with $\varphi_{j} \in \mathscr{R}$.

$\varrho$ is assumed to be stationary under the free dynamics of $S$, and may depend, a priori, on $\mathrm{H}$ and on $\mathrm{gV}$. 
Then, if $\mathscr{R}$ satisfies assumptions a),..., d), there exists a real number $\beta$ such that $\hat{h}_{j i}(-\lambda)=\exp (-\beta \lambda) \hat{h}_{i j}(\lambda)$ for all $\lambda \in \mathbb{R}, \quad \varphi_{i}, \varphi_{j} \in \mathscr{R}$,

and $\varrho$ is the canonical state $\varrho_{\beta}=\exp (-\beta H) / \operatorname{Tr}[\exp (-\beta H)]$ (when $\mathscr{H}$ is infinitedimensional, this forces $\beta$ to be positive). In the quasifree fermion case, or if e) or $\mathrm{e}^{\prime}$ ) holds, $\omega$ is KMS at inverse temperature $\beta$.

Proof. The strategy of the proof consists in showing that the ratio $\hat{h}_{j i}(-\lambda) / \hat{h}_{i j}(\lambda)$ is a positive multiplicative function of $\lambda$, which does not depend on $i$ and $j$, as in [21].

We can assume the spectrum of $H$ to be nondegenerate and fix our attention on six eigenvectors of $H$, which we label $|1\rangle, \ldots,|6\rangle$, with eigenvalues $\varepsilon_{1}, \ldots, \varepsilon_{6}$. Let $\varepsilon_{s} \neq \varepsilon_{s^{\prime}}$ for $s \neq s^{\prime}$ and $\varepsilon_{s+3}-\varepsilon_{s^{\prime}+3}=\varepsilon_{s}-\varepsilon_{s^{\prime}}\left(s, s^{\prime}=1,2,3\right)$. We consider only couplings of the form (3.1), where the operators $F_{j}$ are linear combinations of the $P_{r r^{\prime}}$ with $r, r^{\prime}=1, \ldots, 6$. Then all the summations in the expression (3.2) of $L$ are finite, and we can define a bounded linear operator $L^{\prime}$ on $\mathscr{B}(\mathscr{H})$ by

$$
\operatorname{Tr}\left[\varrho L^{\prime}(A) B\right]=\operatorname{Tr}[\varrho A L(B)], \quad A, B \in \mathscr{B}(\mathscr{H}) .
$$

The most convenient way of imposing detailed balance on $L$ is to require the $\varrho$ antisymmetric part $L_{a}=(1 / 2)\left(L-L^{\prime}\right)$ of $L$ to be a derivation. A straightforward computation yields

$$
\begin{aligned}
L_{a}(A)= & \sum_{\lambda} \sum_{\substack{r r^{\prime} s^{\prime}=1 \\
\varepsilon_{r}-\varepsilon_{r^{\prime}}=\varepsilon_{s}-\varepsilon_{s^{\prime}}=\lambda}}^{6} \sum_{i j=1}^{n}\left(F_{j}\right)_{r r^{\prime}}\left(F_{i}\right)_{s^{\prime} s}\left(i s_{i j}(\lambda) \delta_{r^{\prime} s^{\prime}}\left[P_{r s}, A\right]\right. \\
& \left.+\left[\hat{h}_{j i}(-\lambda)-\varrho_{s} \varrho_{s^{\prime}}^{-1} \hat{h}_{i j}(\lambda)\right] P_{s^{\prime} s} A P_{r r^{\prime}}\right),
\end{aligned}
$$

where $A \in \mathscr{B}(\mathscr{H})$. The condition $L_{a}\left(P_{m n} P_{p q}\right)=L_{a}\left(P_{m n}\right) P_{p q}+P_{m n} L_{a}\left(P_{p q}\right)$ for all $m, n, p$, $q$ is equivalent to the following two equations

$$
\sum_{i j=1}^{n}\left[\hat{h}_{j i}(-\lambda)-\varrho_{s} \varrho_{s^{\prime}}^{-1} \hat{h}_{i j}(\lambda)\right]\left(F_{j}\right)_{r r^{\prime}}\left(F_{i}\right)_{s^{\prime} s}=0
$$

where $r \neq r^{\prime}, s \neq s^{\prime}, \lambda=\varepsilon_{r}-\varepsilon_{r^{\prime}} \neq 0$, and

$$
\sum_{i j=1}^{n}\left[\hat{h}_{j i}(0)-\hat{h}_{i j}(0)\right]\left(F_{j}\right)_{r r}\left(F_{i}\right)_{s s}=0 .
$$

Take $\varphi_{1}, \varphi_{2}$ in $\mathscr{R}$. By choosing a coupling

$$
V=F_{1} \otimes \varphi_{1}+F_{2} \otimes \varphi_{2}
$$

where $F_{1}$ and $F_{2}$ are suitable linear combinations of the $P_{r r^{\prime}}, r, r^{\prime}=1, \ldots, 6$, it can be seen from (3.10) and (3.11) that $\hat{h}_{12}(\lambda)=0$ for some $\lambda$ if and only if $\hat{h}_{21}(-\lambda)=0$.

Let $E$ be the subset of $\mathbb{R}$ on which $\hat{h}_{12}(\lambda)$ does not vanish. By assumptions a), .., d), for any positive integer $m$ it is possible to choose a finite collection $\left\{\varphi_{j}\right\}_{j=3}^{n(m)}$ of operators in $\mathscr{R}$, smeared with test functions whose Fourier transforms vanish in $E$, and such that

$$
\left.\begin{array}{ll}
\sum_{i j=1}^{n(m)} \hat{h}_{i j}(\lambda) \neq 0 \quad \text { for all } & \lambda \in(-m, m), \\
\hat{h}_{i j}(\lambda)=0 \quad \text { for } \quad \lambda \in E, & i, j=3, \ldots, n(m), \\
\hat{h}_{j 1}=\hat{h}_{1 j}=\hat{h}_{j 2}=\hat{h}_{2 j}=0 \quad \text { identically for } j=3, \ldots, n(m) .
\end{array}\right\}
$$


Choose a coupling

$$
V^{(m)}=\sum_{j=1}^{n(m)} F_{j} \otimes \varphi_{j}
$$

with

$$
\begin{aligned}
& F_{1}=\left(P_{12}+P_{23}+P_{31}\right)+\text { h.c., } \quad F_{2}=\left(P_{45}+P_{56}+P_{64}\right)+\text { h.c., } \\
& F_{j}=F_{1}+F_{2} \text { for } j=3, \ldots, n(m),
\end{aligned}
$$

so that Equation (3.10) yields

$$
\hat{h}_{21}(-\lambda)+\sum_{i j=3}^{n(m)} \hat{h}_{j i}(-\lambda)=\varrho_{s} \varrho_{s^{\prime}}^{-1}\left[\hat{h}_{12}(\lambda)+\sum_{i j=3}^{n(m)} \hat{h}_{i j}(\lambda)\right]
$$

for all $s, s^{\prime}=1,2,3, r=s+3, r^{\prime}=s^{\prime}+3, \lambda=\varepsilon_{s}-\varepsilon_{s^{\prime}} \neq 0$. Define a function $\mu^{(m)}(\lambda)$ on $(-m, m)$, with values in $(0, \infty)$, by

$$
\mu^{(m)}(\lambda)=\left[\hat{h}_{21}(-\lambda)+\sum_{i j=3}^{n(m)} \hat{h}_{j i}(-\lambda)\right]\left[\hat{h}_{12}(\lambda)+\sum_{i j=3}^{n(m)} \hat{h}_{i j}(\lambda)\right]^{-1} .
$$

By Equation (3.13), $\mu^{(m)}(\lambda)=\varrho_{s} \varrho_{s^{\prime}}^{-1}\left(\varepsilon_{s}-\varepsilon_{s^{\prime}}=\lambda\right)$ for $\lambda \neq 0$.

It follows that

$$
\mu^{(m)}(\lambda) \mu^{(m)}\left(\lambda^{\prime}\right)=\mu^{(m)}\left(\lambda+\lambda^{\prime}\right)
$$

for $\lambda, \lambda^{\prime}, \lambda+\lambda^{\prime}$ in $(-m, m)$ and different from zero. By the continuity of $\mu^{(m)}(\lambda)$, this still holds when some of the $\lambda, \lambda^{\prime}, \lambda+\lambda^{\prime}$ are zero.

If $m<m^{\prime}$, by suitably smearing the operators $\varphi_{j}\left(n(m)<j \leqq n\left(m^{\prime}\right)\right)$ with test functions having energy support disjoint from $(-m, m)$, we can fulfill the condition

$$
\mu^{(m)}(\lambda)=\mu^{\left(m^{\prime}\right)}(\lambda) \text { for }|\lambda|<m .
$$

Therefore, the limit of the sequence $\left\{\mu^{(m)}\right\}$ defines a function $\mu: \mathbb{R} \rightarrow(0, \infty)$ which satisfies

$$
\mu(\lambda) \mu\left(\lambda^{\prime}\right)=\mu\left(\lambda+\lambda^{\prime}\right) \text { for all } \lambda, \lambda^{\prime} \quad \text { in } \mathbb{R} .
$$

Hence $\mu(\lambda)$ is an exponential. For $\lambda$ in $E, \mu(\lambda)$ reduces to $\hat{h}_{21}(-\lambda) / \hat{h}_{12}(\lambda)$ by Equation (3.12). Hence

$$
\hat{h}_{21}(-\lambda)=\exp \left(-\beta_{12} \lambda\right) \hat{h}_{12}(\lambda)
$$

for some real number $\beta_{12}$.

We show that $\beta_{12}$ is independent of the choice of $\varphi_{1}, \varphi_{2}$ in $\mathscr{R}$. By assumption d), we can find a positive number $M$ and two elements $\varphi_{1}, \varphi_{2}$ of $\mathscr{R}$ such that

$$
\hat{h}_{12}(\lambda) \neq 0 \text { for all }|\lambda|<M,
$$

and a pair $\varphi_{3}, \varphi_{4}$ in $\mathscr{R}$ such that $\hat{h}_{34}\left(\lambda_{0}\right) \neq 0$ for some $\lambda_{0} \in(-2 M, 2 M)$. Choose a coupling

$$
V=\sum_{j=1}^{4} F_{j} \otimes \varphi_{j}
$$


with

$$
\begin{array}{ll}
F_{1}=\left(P_{12}+P_{23}\right)+\text { h.c., } & F_{2}=\left(P_{45}+P_{56}\right)+\text { h.c., } \\
F_{3}=P_{13}+\text { h.c. } & F_{4}=P_{46}+\text { h.c. }
\end{array}
$$

Then, setting $\varepsilon_{1}-\varepsilon_{3}=\lambda_{0}$ and using Equation (3.16), Equation (3.10) yields

$$
\begin{aligned}
& \exp \left(-\beta_{12} \lambda\right)=\varrho_{1} \varrho_{2}^{-1} \quad\left(\lambda=\varepsilon_{1}-\varepsilon_{2}\right), \\
& \exp \left(-\beta_{12} \lambda^{\prime}\right)=\varrho_{2} \varrho_{3}^{-1} \quad\left(\lambda^{\prime}=\varepsilon_{2}-\varepsilon_{3}\right), \\
& \exp \left(-\beta_{34}\left(\lambda+\lambda^{\prime}\right)\right)=\varrho_{1} \varrho_{3}^{-1},
\end{aligned}
$$

so that $\beta_{34}=\beta_{12}$. This proves that the real number $\beta$ is the same for all correlation functions whose Fourier transforms do not vanish identically in $(-2 M, 2 M)$. Hence, using again assumption d), we can choose a finite collection $\left\{\varphi_{j}\right\}_{j=1}^{p}$ such that

$$
\sum_{i j=1}^{p} \hat{h}_{i j}(\lambda) \neq 0 \text { for all }|\lambda|<2 M
$$

and

$$
\hat{h}_{j i}(-\lambda)=\exp (-\beta \lambda) \hat{h}_{i j}(\lambda) \text { for all } i, j=1, \ldots, p .
$$

Then, let $\varphi_{q}, \varphi_{r}$ in $\mathscr{R}$ be such that $\hat{h}_{q r}\left(\lambda_{0}\right) \neq 0$ for some $\lambda_{0} \in(-4 M, 4 M)$, and choose a coupling

$$
V=\sum_{j=1}^{r} F_{j} \otimes \varphi_{j}
$$

with

$$
\begin{aligned}
& F_{j}=\left(P_{12}+P_{23}+P_{45}+P_{56}\right)+\text { h.c. for } j=1, \ldots, p, \\
& F_{q}=P_{13}+\text { h.c., } \quad F_{r}=P_{46}+\text { h.c. }
\end{aligned}
$$

Then, setting $\varepsilon_{1}-\varepsilon_{3}=\lambda_{0}$ and using Equation (3.17), Equation (3.10) yields

$$
\begin{aligned}
& \exp (-\beta \lambda)=\varrho_{1} \varrho_{2}^{-1} \quad\left(\lambda=\varepsilon_{1}-\varepsilon_{2}\right), \\
& \exp \left(-\beta \lambda^{\prime}\right)=\varrho_{2} \varrho_{3}^{-1} \quad\left(\lambda^{\prime}=\varepsilon_{2}-\varepsilon_{3}\right), \\
& \exp \left(-\beta_{q r}\left(\lambda+\lambda^{\prime}\right)\right)=\varrho_{1} \varrho_{3}^{-1} .
\end{aligned}
$$

Hence $\beta_{q r}=\beta$. Iterating the above argument proves that there exists a real number $\beta$ such that Equation (3.6) holds for all $\varphi_{i}, \varphi_{j}$ in $\mathscr{R}$.

From the above discussion, it follows that the eigenvalues $\varrho_{r}$ of $\varrho(r=1, \ldots, 6)$ are given by

$$
\varrho_{r}=\exp \left[-\beta\left(\varepsilon_{r}-\varepsilon_{1}\right)\right] \varrho_{1} .
$$

Since the choice of the six eigenvectors $|1\rangle, \ldots,|6\rangle$ is arbitrary, Equation (3.18) holds for all the eigenvalues of $\varrho$. Then, $\varrho$ is proportional to $\exp (-\beta H)$. If $\mathscr{H}$ is infinitedimensional, this rules out the case $\beta \leqq 0$. 
In the quasifree fermion case, the KMS condition (3.17) can be extended to the strongly dense *-algebra of polynomials in the operators of $\mathscr{R}$, using the expansion formulas

$$
\begin{aligned}
& \omega\left(\alpha_{t_{1}}\left(\varphi_{j_{1}}\right) \ldots \alpha_{t_{2 n+1}}\left(\varphi_{j_{2 n+1}}\right)\right)=0, \\
& \omega\left(\alpha_{t_{1}}\left(\varphi_{j_{1}}\right) \ldots \alpha_{t_{2 n}}\left(\varphi_{j_{2 n}}\right)=\sum_{p \in \mathscr{P}_{n}} \operatorname{sign} p \prod_{r=1}^{n} \omega\left(\alpha_{t_{p(2 r-1)}}\left(\varphi_{j_{p(2 r-1)}}\right) \alpha_{t_{p(2 r)}}\left(\varphi_{\left.j_{p(2 r}\right)}\right),\right.\right.
\end{aligned}
$$

where $\mathscr{P}_{n}$ is the set of those permutations $p$ of $\{1, \ldots, 2 n\}$ for which $p(2 r-1)<p(2 r)$ and $p(2 r-1)<p(2 r+1)$. The extension to the whole $\mathscr{A}_{\omega}$ follows from Corollary 1 , p. 200, of [22]. The same Corollary allows the extension of the KMS condition to the whole algebra for general reservoirs, under assumption e). When e') holds, the extension can be made with the same arguments as in [21]. Q.E.D.

The formal similarity of the above proof with the arguments of [21], in which the KMS condition was derived as a consequence of stability, suggests the existence of a link between stability and detailed balance. This is confirmed by the following

Theorem 3.2. Let $R=\left(\mathscr{A}, \omega, \alpha_{t}\right)$, and let $S$ be a spatially confined quantum system whose free Hamiltonian $H$ possesses a nondegenerate spectrum. Assume that the reduced dynamics of $S$ derived in the limit of weak coupling to $R$ leaves invariant a faithful state $\varrho$, which does not depend on the coupling $g V$ and is stationary under the free dynamics of $S$. Then the reduced dynamics $\{\exp (L t)\}$ of $S$ satisfies detailed balance w.r.t. $\varrho$.

Proof. Since the spectrum of $H$ is nondegenerate, $\varrho$ commutes with $H$ as well as with the Hamiltonian part of the reduced dynamics. Hence, it is a stationary state for the reduced dynamics iff

$$
\begin{gathered}
\sum_{\substack{r r^{\prime} s s^{\prime} \\
\varepsilon_{r}-\varepsilon_{r^{\prime}}=\varepsilon_{s}-\varepsilon_{s^{\prime}}}} \sum_{i j=1}^{n} \hat{h}_{i j}\left(\varepsilon_{r}-\varepsilon_{r^{\prime}}\right)\left(F_{j}\right)_{r r^{\prime}}\left(F_{i}\right)_{s^{\prime} s} \\
\cdot\left(P_{s^{\prime} s} \varrho P_{r r^{\prime}}-\frac{1}{2} P_{r r^{\prime}} P_{s^{\prime} s} \varrho-\frac{1}{2} \varrho P_{r r^{\prime}} P_{s^{\prime} s}\right)=0 .
\end{gathered}
$$

Now, $\varrho P_{r r^{\prime}}=\varrho_{r} P_{r r^{\prime}}$. With $r=s, r^{\prime}=s^{\prime}$, the above formula yields

$$
\sum_{r^{\prime}} \sum_{i j=1}^{n}\left(F_{j}\right)_{r r^{\prime}}\left(F_{i}\right)_{r^{\prime} r}\left[\hat{h}_{i j}\left(\varepsilon_{r}-\varepsilon_{r^{\prime}}\right) \varrho_{r}-\hat{h}_{j i}\left(\varepsilon_{r^{\prime}}-\varepsilon_{r}\right) \varrho_{r^{\prime}}\right]=0
$$

for all $r$. Fix two indices $k, k^{\prime}\left(k \neq k^{\prime}\right)$ and let $F_{j}=\lambda_{j}\left(P_{k k^{\prime}}+P_{k^{\prime} k}\right)+i \mu_{j}\left(P_{k k^{\prime}}-P_{k^{\prime} k}\right), \lambda_{j}$, $\mu_{j} \in \mathbb{R}$. Then Equation (3.20) with $r=k$ gives $\left(z_{j}=\lambda_{j}+i \mu_{j}\right)$

$$
\sum_{i j=1}^{n} \overline{z_{j}}\left[\hat{h}_{i j}\left(\varepsilon_{k}-\varepsilon_{k^{\prime}}\right) \varrho_{k}-\hat{h}_{j i}\left(\varepsilon_{k^{\prime}}-\varepsilon_{k}\right) \varrho_{k^{\prime}}\right] z_{j}=0 .
$$

The expression within square brackets defines a Hermitian matrix for all $k, k^{\prime}$. The complex numbers $z_{j}$ are arbitrary, hence

$$
\hat{h}_{j i}\left(\varepsilon_{k^{\prime}}-\varepsilon_{k}\right)=\varrho_{k} \varrho_{k^{\prime}}^{-1} \hat{h}_{i j}\left(\varepsilon_{k}-\varepsilon_{k^{\prime}}\right) \quad\left(k \neq k^{\prime}\right) .
$$

Upon inserting Equation (3.21) into Equation (3.20), it is seen that (3.21) holds also for $k=k^{\prime}$. Thus the non-Hamiltonian part of $L$ is $\varrho$-symmetric. Its Hamiltonian part is clearly $\varrho$-antisymmetric. This amounts to detailed balance. Q.E.D. 
Remark. The hypothesis of Theorem 3.2 is a stability property of the state $\varrho \otimes \omega$ of the combined system $S+R$, and it can be regarded as a natural characterization of what is meant by saying that the system $S$ is in thermal equilibrium with the "heat bath" $R$ (compare [23]).

\section{Appendix A}

Uniqueness of the Decomposition (2.2)

Suppose that

$$
\hat{L} \psi=\hat{L}_{s} \psi+\hat{L}_{h} \psi \text { for all } \psi \text { in a common core } \mathscr{C} \text { for } \hat{L}, \hat{L}_{s}, \hat{L}_{h}
$$
and

$$
\hat{L} \psi^{\prime}=\hat{L}_{s}^{\prime} \psi^{\prime}+\hat{L}_{h}^{\prime} \psi^{\prime} \text { for all } \psi^{\prime} \text { in a common core } \mathscr{C}^{\prime} \text { for } \hat{L}, \hat{L}_{s}^{\prime}, \hat{L}_{h}^{\prime},
$$

where $\hat{L}_{s}, \hat{L}_{s}^{\prime}$ are selfadjoint and $\hat{L}_{h}, \hat{L}_{h}^{\prime}$ are skewadjoint. Let $\hat{s}$ be the symmetric semibounded sesquilinear form defined on $\mathscr{D}(\hat{L})$ by

$$
\hat{s}(\phi, \psi)=\frac{1}{2}[(\phi \mid \hat{L} \psi)+\overline{(\psi \mid \hat{L} \phi)}], \quad \phi, \psi \in \mathscr{D}(\hat{L}),
$$

and let $s$ (resp., $s^{\prime}$ ) be the restriction of $\hat{s}$ to $\mathscr{C}$ (resp., $\mathscr{C}^{\prime}$ ). Then

$$
\begin{array}{rlrl}
s(\phi \mid \psi) & =\left(\phi \mid \hat{L}_{s} \psi\right) & & \varphi, \psi \in \mathscr{C}, \\
s^{\prime}\left(\phi^{\prime} \mid \psi^{\prime}\right) & =\left(\phi^{\prime} \mid \hat{L}_{s}^{\prime} \psi^{\prime}\right) & \phi^{\prime}, \psi^{\prime} \in \mathscr{C}^{\prime} .
\end{array}
$$

Clearly $s$ and $s^{\prime}$ are closable. Moreover, they are contained in the closure of each other. Indeed, since $\mathscr{C}$ is a core for $\hat{L}$ and $\mathscr{C}^{\prime} \subset \mathscr{D}(\hat{L})$, for all $\psi^{\prime} \in \mathscr{C}^{\prime}$ there exists $\left\{\psi_{n}\right\} \subset \mathscr{C}$ such that $\psi_{n} \rightarrow \psi^{\prime}$ and $\hat{L} \psi_{n} \rightarrow \hat{L} \psi^{\prime}$. Then

$$
\begin{aligned}
\left|s\left(\psi_{n}-\psi_{m} \mid \psi_{n}-\psi_{m}\right)\right| & =\left|\operatorname{Re}\left(\psi_{n}-\psi_{m} \mid \hat{L}\left(\psi_{n}-\psi_{m}\right)\right)\right| \\
& \leqq\left\|\psi_{n}-\psi_{m}\right\|\left\|\hat{L} \psi_{n}-\hat{L} \psi_{m}\right\| \rightarrow 0
\end{aligned}
$$

Hence $\psi^{\prime}$ is in the domain of the closure of $s$. The same argument can be repeated with the interchange of $\mathscr{C}$ and $\mathscr{C}^{\prime}$. Then the closure of $s$ and the closure of $s^{\prime}$ coincide. Thus $\hat{L}_{s} \uparrow \mathscr{C}$ and $\hat{L}_{s}^{\prime} \uparrow \mathscr{C}^{\prime}$ have the same Friedrichs extension [24]. But since they are essentially selfadjoint, $\hat{L}_{s}=\hat{L}_{s}^{\prime}$. Finally, the closure of $\hat{L}-\hat{L}_{s}$ is a skewsymmetric closed extension of both $\hat{L}_{h} \uparrow \mathscr{C}$ and $\hat{L}_{h}^{\prime} \uparrow \mathscr{C}^{\prime}$. Hence $\hat{L}_{h}=\hat{L}_{h}^{\prime}=\hat{L}-\hat{L}_{s}$.

\section{Appendix B}

\section{Proof of Equation (2.4)}

Let $\hat{U}_{t}=\exp \left(\hat{L}_{h} t\right), \hat{\Gamma}_{t}=\exp \left(\hat{L}_{s} t\right)$. Let $\left\{\Lambda_{t}\right\}$ (resp., $\left.\left\{V_{t}\right\}\right)$ be the strongly continuous predual semigroup of $\left\{\Phi_{t}\right\}$ (resp., predual group of $\left\{\alpha_{t}\right\}$ ), and let $\mathscr{L}$ (resp., $\mathscr{L}_{h}$ ) be its generator. Define $\mathscr{L}_{s}$ as the closure of $\mathscr{L}-\mathscr{L}_{h}$. For $\psi \in \mathscr{K}$, define $\varrho_{\psi} \in \mathscr{M}_{*}$ by $\varrho_{\psi}(A)$ $=(\psi \mid \pi(A) \Omega)$ for all $A \in \mathscr{M}$. The linear manifold $\mathscr{E}=\left\{\varrho_{\psi}: \psi \in \mathscr{C}\right\}$ is in the domain of $\mathscr{L}_{s}$ and

$$
\mathscr{L}_{s} \varrho_{\psi}=\varrho_{\hat{L}_{s} \psi} \text { for all } \psi \in \mathscr{C} .
$$

The manifolds $\mathscr{E}$ and $\left(\mathscr{I}-\mathscr{L}_{s}\right) \mathscr{E}$ (where $\mathscr{I}$ denotes the identity map of $\mathscr{M}_{*}$ ) are dense in $\mathscr{M}_{*}$. Indeed, $\mathscr{C}$ and $\left(\mathbb{1}-\hat{L}_{s}\right) \mathscr{C}$ are dense in $\mathscr{K}$ since $\mathscr{C}$ is a core for $\hat{L}_{s}$, the range of $\left(\mathbb{1}-\mathscr{L}_{s}\right)$ is $\mathscr{K}$, and the set $\mathscr{S}=\left\{\varrho_{\psi}: \psi \in \mathscr{K}\right\}$ is dense in $\mathscr{M}_{*}$ by [25, Example 5]. 
But since $\mathscr{L}_{s}$ is dissipative, $\left(\mathscr{I}-\mathscr{L}_{s}\right)^{-1}$ exists and is continuous, hence the range of $\left(\mathscr{I}-\mathscr{L}_{s}\right)$ is the whole of $\mathscr{M}_{*}$. Then $\mathscr{L}_{s}$ is the generator of a strongly continuous contraction semigroup $\left\{Y_{t}\right\}$ of $\mathscr{M}_{*}$, by the Phillips-Lumer theorem. $Y_{t}$ is given by the Trotter product formula

$$
Y_{t}=s-\lim _{n \rightarrow \infty}\left(V_{-t / n} \Lambda_{t / n}\right)^{n}
$$

Passing to the dual maps,

$$
Y_{t}^{*}(A)=w_{n \rightarrow \infty}^{*-\lim }\left(\Phi_{t / n} \alpha_{-t / n}\right)^{n}(A) \text { for all } A \in \mathscr{M} .
$$

It can be easily verified that $\left\{Y_{t}^{*}\right\}$ is a dynamical semigroup of $\mathscr{M}$. In particular, complete positivity follows from the fact that the cone of completely positive maps of a $W^{*}$-algebra is closed in the bounded-weak topology [16]. For all $\psi \in \mathscr{K}, A \in \mathscr{M}$, we have

$$
\begin{aligned}
\left(\psi \mid \hat{\Gamma}_{t} \pi(A) \Omega\right) & =\lim _{n \rightarrow \infty}\left(\psi \mid\left(\hat{\Phi}_{t / n} \hat{U}_{-t / n}\right)^{n} \pi(A) \Omega\right) \\
& =\lim _{n \rightarrow \infty}\left(\psi \mid \pi\left(\left(\Phi_{t / n} \alpha_{-t / n}\right)^{n}(\mathrm{~A})\right) \Omega\right) \\
& =\left(\psi \mid \pi\left(Y_{t}^{*}(A)\right) \Omega\right)
\end{aligned}
$$

which proves (2.4) with $Y_{t}^{*}=\Gamma_{t}$.

\section{Appendix C}

Proof of (2.16)-(2.20)

Starting from the form (2.8) of $L_{s}$, we can write $L_{s}=L_{1}+L_{2}$, where

$$
\begin{aligned}
& L_{1}(A)=\sum_{\substack{r r^{\prime} s s^{\prime}=1 \\
\varrho r=\varrho r^{\prime}, \varrho_{s}=\varrho_{s^{\prime}}}}^{N} C_{r r^{\prime} s s^{\prime}}\left[P_{r r^{\prime}} A P_{s^{\prime} s}-\frac{1}{2}\left\{P_{r r^{\prime}} P_{s^{\prime} s}, A\right\}\right], \\
& L_{2}(A)=\sum_{\substack{r r^{\prime} s s^{\prime}=1 \\
\varrho_{r} \neq \varrho_{r^{\prime}}, \varrho_{s} \neq \varrho_{s^{\prime}}}} C_{r r^{\prime} s s^{\prime}}\left[P_{r r^{\prime}} A P_{s^{\prime} s}-\frac{1}{2}\left\{P_{r r^{\prime}} P_{s^{\prime} s}, A\right\}\right] .
\end{aligned}
$$

$L_{1}$ is a $\varrho$-symmetric and $\tau$-symmetric generator, hence it can be given the form (2.15), which in turn can be diagonalized in terms of nonnegative coefficients $D_{i j}$ and selfadjoint operators satisfying (2.17). From the $\varrho$-symmetry and $\tau$-symmetry of $L_{1}$ it follows $\varrho L_{1}(A)=L_{1}(\varrho A)$ and $L_{1}(A) \varrho=L_{1}(A \varrho)$ for all $A$ in $\mathscr{B}(\mathscr{H})$, hence, by [26], $\varrho$ commutes with the operators $X_{i j}$ appearing in $L_{1}$. As for $L_{2}$, it is a $\varrho$-symmetric generator to which the arguments of [3] can be applied (this is indeed the major part of the proof). The result then follows.

\section{References}

1. Agarwal,G.S.: Z. Physik 258, 409-422 (1973)

2. Carmichael,H.J., Walls,D. F.: Z. Physik B 23, 299-306 (1976)

3. Alicki, R.: Rep. Math. Phys. 10, 249-258 (1976)

4. Gorini,V., Frigerio, A., Verri,M., Kossakowski,A., Sudarshan,E.C.G.: Properties of quantum markovian master equations. Rep. Math. Phys. (to appear) 
5. Ingarden, R. S., Kossakowski, A.: Ann. Phys. (N.Y.) 89, 451-485 (1975)

6. Gorini,V., Kossakowski, A., Sudarshan,E.C.G.: J. Math. Phys. 17, $821-825$ (1976)

7. Lindblad, G.: Commun. math. Phys. 48, 119-130 (1976)

8. Kossakowski, A.: Bull. Acad. Polon. Sci. Math. (to appear)

9. Evans, D.E., Lewis,J.T.: Completely positive maps on the CCR algebras. J. Funct. Anal. (to appear)

10. Takesaki,M.: Tomita's theory of modular Hilbert algebras and its applications. Lecture notes in mathematics, Vol. 128. Berlin-Heidelberg-New York: Springer 1970

11. Sirugue, M., Winnink,M.: Commun. math. Phys. 19, 161-168 (1970)

12. Bratteli,O., Robinson,D.W.: Ann. Inst. H.Poincaré 25, 139-164 (1976)

13. Sakai,S.: $C^{*}$-algebras and $W^{*}$-algebras. Berlin-Heidelberg-New York: Springer 1971

14. Reed,M., Simon, B. : Methods of modern mathematical physics. Vol. II. New York: Academic Press 1975

15. Davies, E. B. : Commun. math. Phys. 49, 113-129 (1976)

16. Arveson, W.B.: Acta Math. 123, 141-224 (1969)

17. Kraus, K.: Ann. Phys. (N.Y.) 64, 311-335 (1971)

18. Gorini,V., Kossakowski, A.: J. Math. Phys. 17, 1298-1305 (1976)

19. Frigerio, A., Gorini,V.: J. Math. Phys. 17, 2123-2127 (1976)

20. Davies, E. B.: Commun. math. Phys. 39, 91-110 (1974)

21. Haag, R., Kastler,D., Trych-Pohlmeyer,E.: Commun. math. Phys. 38, 173-193 (1974)

22. Emch,G.G.: Algebraic methods in statistical mechanics and quantum field theory. New York: Wiley-Interscience 1972

23. Sewell,G.L.: Ann. Phys. (N.Y.) 85, 336-377 (1974)

24. Kato,T.: Perturbation theory for linear operators. Berlin-Heidelberg-New York: Springer 1966

25. Radin, C.: Commun. math. Phys. 21, 291-302 (1971)

26. Evans, D.E.: Commun. math. Phys. 54, 293-297 (1977)

Communicated by H. Araki

Received March 22, 1977; in revised form June 13, 1977 